\title{
Violência Física Doméstica e Gestação: Resultados de um Inquérito no Puerpério
}

\author{
Domestic Physical Violence and Pregnancy: \\ Results of a Survey in the Postpartum Period
}

Telma Cursino Menezes, Melania Maria Ramos de Amorim Luiz Carlos Santos, Aníbal Faúndes

\section{RESUMO}

Objetivos: determinar a prevalência de violência fisica doméstica sofrida por mulheres que tiveram o parto assistido em uma maternidade terciária do Nordeste do Brasil, estudar os principais fatores de risco associados e determinar os resultados perinatais.

Métodos: realizou-se estudo descritivo, tipo corte transversal, incluindo 420 mulheres que tiveram o parto assistido em maternidade terciária no Recife (Brasil), com fetos pesando mais que 500 gramas. Todas estas foram submetidas a entrevistas com questões abertas e fechadas. Determinou-se a prevalência de violência física doméstica. Realizou-se análise estatística usando os testes $\chi^{2}$ de associação e exato de Fisher, considerando-se o nivel de significância de 5\%. A razão de prevalência foi determinada como medida do risco relativo de violência. Realizou-se análise de regressão logística para cálculo do risco ajustado de violência física. Resultados: a prevalência de violência fisica doméstica foi de 13,1\% (IC 95\% = 10,1-16,6) $e$ $7,4 \%(I C 95 \%=5,2-10,2)$ antes e durante a gestação, respectivamente. O padrão de violência alterou-se durante a gravidez, tendo cessado em 43,6\%, diminuido em 27,3\% e aumentado em $11 \%$ dos casos. Depois da análise multivariada, as variáveis que persistiram fortemente associadas com violência foram baixa escolaridade e história familiar de violência da mulher, consumo de álcool e desemprego do parceiro. Entre os resultados perinatais, encontrou-se elevada freqüência de morte neonatal entre as vitimas de violência.

Conclusões: encontrou-se elevada prevalência de violência física doméstica (cerca de 13\%) entre as mulheres que tiveram o parto assistido em maternidade terciária do Nordeste do Brasil. Os principais fatores de risco associados foram baixa escolaridade e história de violência na família da mulher, consumo de álcool e desemprego dos parceiros. A mortalidade neonatal foi elevada entre as vitimas de violência.

PALAVRAS-CHAVE: Violência contra a mulher. Violência doméstica. Gravidez. Violência e gravidez: fatores de risco.

\section{Introdução}

De acordo com a OMS, a violência representa problema de saúde pública de graves dimensões, amplamente disseminado em todos os países do mundo. Nessa óptica, a violência contra a mulher

Instituto Materno-Infantil de Pernambuco (IMIP) - Mestrado em Saúde Materno-Infantil - Recife/PE

Correspondência:

Melania Maria Ramos de Amorim

Rua Neuza Borborema de Sousa, 300 - Bairro Santo Antônio

58103-313 - Campina Grande - PB

e-mail: melamorim@uol.com.br assume especial relevância, estimando-se que pelo menos um quinto da população feminina mundial tenha já sofrido violência física ou sexual em algum momento de suas vidas ${ }^{1}$.

Sob a epígrafe "violência contra a mulher" encontra-se um leque de situações as mais diversas, incluindo tanto violência física como sexual e emocional. A definição da Assembléia Geral das Nações Unidas, em 1993, reconhece como violência contra a mulher "qualquer ato de violência de gênero que resulte, ou tenha probabilidade de resultar, em prejuízo físico, sexual ou psicológico, ou ainda sofrimento para as mulheres, 
incluindo também a ameaça de praticar tais atos, a coerção e a privação da liberdade, ocorrendo tanto em público como na vida privada" ${ }^{\text {. }}$.

O termo violência doméstica tem sido utilizado para se referir a todas as formas de violência praticada no ambiente familiar ${ }^{2}$, porém reflete, geralmente, a violência contra a mulher perpetrada por seu parceiro íntimo ${ }^{3}$.

Presente na maioria das sociedades, a violência praticada pelo parceiro íntimo constitui a forma mais endêmica de violência contra a mu1 lher ${ }^{1,4}$, no entanto não é reconhecida como forma de violência, sendo muitas vezes aceita como fenômeno cultural ${ }^{1}$, fazendo parte dos costumes e normas da sociedade que entendem e aceitam a violência exercida contra mulheres como forma de ação disciplinar exercida sobre esposas e filhas ${ }^{5}$. Segundo estimativas do Banco Mundial, uma mulher tem maior probabilidade de ser espancada, violada ou assassinada pelo seu parceiro atual ou anterior que por um estranho ${ }^{1,5}$.

A real extensão da violência doméstica é ainda difícil de ser averiguada, em razão de variações metodológicas quanto à definição de violência, tamanho amostral e metodologia de inquérito ${ }^{6,7}$. Além disso, os profissionais de saúde não estão em geral habilitados para diagnosticar a presença de violência doméstica, e dificilmente irão introduzir perguntas sobre o assunto durante a anamnese. No estudo de Rodriguez et al. ${ }^{8}$, apenas 9 a $11 \%$ dos médicos investigaram sobre VD em pacientes procurando serviços de assistência primária à saúde 8 .

Estima-se que 20 a $30 \%$ de todas as mulheres americanas tenham sido vítimas de violência por um parceiro íntimo ao longo de suas vidas ${ }^{9} \mathrm{e}$ que cerca de três quartos das mulheres americanas que sofreram agressão foram vitimas de seus parceiros $^{10}$.

Embora não sejam dados conclusivos, estudos apontam a gravidez como fator de risco para a violência doméstica ${ }^{5}$, podendo esta ter início depois da gestação ou alterar o padrão quanto à freqüência e gravidade neste periodo ${ }^{11}$. Estudos de revisão sobre prevalência de VD na gravidez indicam uma estimativa de 0,9 a $20,1 \%^{12-14}$, referindo a maioria dos estudos taxas entre 3,9 e $8,3 \%$ entre mulheres grávidas investigadas ${ }^{15,16}$.

Diversos danos à saúde podem resultar da $\mathrm{VD}$, variando desde queixas ginecológicas e da esfera sexual ${ }^{17}$ até conseqüências obstétricas diversas como gestações indesejadas ${ }^{15}$, retardo em iniciar o pré-natal ${ }^{15,18,19}$, abortamento e natimortalidade ${ }^{20}$, baixo peso ao nascer ${ }^{19}$, trabalho de parto prematuro e perdas fetais ${ }^{21,22}$. Também podem estar presentes dor pélvica crônica, cefaléia, doença espástica dos cólons ${ }^{23}$, depressão, tentativa de suicídio e síndrome de estresse póstraumático ${ }^{1}$, ansiedade e uso de drogas ${ }^{5}$.

Por outro lado, as gestantes submetidas a violência doméstica podem também ser vitimas de homicídio. Na verdade, o trauma representa causa importante de morte materna em diversos países, sendo 36 a $63 \%$ destas mortes representadas por homicídios, a maioria dos quais praticados pelos parceiros intimos ${ }^{24}$.

Quaisquer medidas de intervenção voltadas para esse problema exigem, como primeiro passo, a identificação das vítimas e, em segundo lugar, a compreensão dos possiveis fatores de risco associados.

No Centro de Atenção à Mulher do Instituto Materno-Infantil de Pernambuco, a investigação de violência não faz parte do protocolo de assistência pré-natal e há necessidade de se conhecer a situação da violência contra as mulheres que recebem assistência na instituição e suas repercussões em alguns parâmetros do bem-estar materno e neonatal. A identificação das condições em que a violência ocorre, suas características e repercussões permitirá ações que promovam sua prevenção e minimização dos agravos.

O presente estudo teve por objetivo determinar a prevalência de violência doméstica e os principais fatores de risco associados em mulheres que receberam assistência ao parto em uma maternidade terciária no Nordeste do Brasil, avaliando ainda os resultados perinatais.

\section{Métodos}

Realizou-se estudo descritivo, tipo corte transversal, com 420 mulheres que tiveram o parto assistido no Centro de Atenção à Mulher (CAM) do Instituto Materno-Infantil de Pernambuco (IMIP), em Recife, PE, nos meses de fevereiro e março de 2001. Essas mulheres corresponderam a $81 \%$ do total de partos assistidos no período, extrapolando o tamanho da amostra originalmente calculado de 380 , prevendo-se prevalência de violência doméstica de $10 \%$, para precisão de $3 \%$ e nível de significância menor que 5\%.

Os critérios de inclusão foram: idade gestacional superior a 24 semanas completas ou recém-nascido com mais de 500 gramas e relacionamento com parceiro íntimo no último ano, independente de coabitação. Excluíram-se as mulheres com condições fisicas ou mentais impedindo a resposta à entrevista, aquelas que se recusaram a participar da pesquisa e os casos de gravidez atual decorrente de violência.

A entrevista foi realizada no puerpério por entrevistadoras treinadas, utilizando questioná- 
rio pré-testado, constituído de questões abertas e fechadas. O questionário foi elaborado utilizando como base instrumento já validado e traduzido para o português, o "Abuse Assessment Screen" - AAS ${ }^{25}$, e que foi aplicado verbalmente, com caráter sigiloso e não judicioso, respeitando as questões culturais.

A análise estatística foi realizada com os programas Epi-Info 2000 e SPSS 8.0 para Windows. $\mathrm{Na}$ primeira etapa da análise, determinou-se a prevalência da violência física no último ano e durante a gravidez, identificando-se as principais formas de agressão e as modificações do padrão de violência durante a gravidez (cessou, diminuiu, aumentou, permaneceu inalterado). Para as finalidades do presente estudo, definiu-se como violência física o uso intencional de força física com potencial para determinar morte, incapacidade, injúria e ferimento, que inclui mas não está limitado a arranhões, bofetadas, empurrões, perfurações, chutes, contusões, queimaduras, fraturas ósseas, espancamento, lesões na cabeça, lesões internas, lesões permanentes e uso de arma (revólver, faca ou outro objeto) ${ }^{1}$.

Na segunda etapa, determinou-se a associação entre violência física (variável dependente) e os possiveis fatores de risco (variáveis independentes), incluindo variáveis ligadas à mulher e ao parceiro, como idade (adolescentes / não adolescentes), escolaridade (anos estudados), situação marital (sem companheiro/união consensual/ casamento civil), história familiar de violência e trabalho remunerado. Estudou-se ainda a história de tabagismo da mulher (fumou ou não durante a gravidez) e consumo de álcool pelo homem (determinado pela freqüência de embriaguez como: nunca, uma vez por mês, uma vez por semana e mais de uma vez por semana). Determinou-se a razão de prevalência como medida do risco relativo de violência física para cada uma dessas variáveis, adotando-se o intervalo de confiança a 95\% (IC 95\%). À categoria de referência atribuiu-se o risco padrão de 1,0. Realizou-se análise de regressão logística condicional (stepwise) para determinação do risco ajustado depois do controle dos fatores potencialmente confundidores. Para essa análise as variáveis foram codificadas binariamente $(\operatorname{sim}=1 /$ não $=0)$.

Finalmente, determinaram-se os resultados perinatais (variáveis dependentes): prematuridade (abaixo de 37 semanas de idade gestacional), baixo peso ao nascer (abaixo de 2.500 gramas), escores de Apgar no primeiro e quinto minutos, morte intra-uterina (feto morto) e morte neonatal precoce (óbito de recém-nascido até 7 dias de vida), de acordo com a presença ou não de violência doméstica, que nessa etapa passou a ser considerada como variável independente, utilizando-se os testes $\chi^{2}$ de associação e exato de Fisher, quando necessário, ao nível de significância de $5 \%$.

O presente estudo respeitou as normas da Declaração de Helsinque e da resolução no 196/96 do Conselho Nacional de Saúde para pesquisa em seres humanos. O projeto que deu origem à pesquisa foi aprovado pelo Comitê de Ética em Pesquisa do IMIP e pela Coordenação Médica do CAM. As entrevistas só puderam ser realizadas mediante aprovação escrita de cada paciente e o caráter sigiloso foi obrigatório.

Quando se encontrou relato de violência fisica contra qualquer das mulheres entrevistadas, ofereceu-se a cada uma destas o acompanhamento pelos serviços de Psicologia e Assistência Social da Instituição. Embora oferecido oportunamente, nenhuma das entrevistadas manifestou desejo de notificar a violência.

\section{Resultados}

Encontrou-se prevalência de $13,1 \%$ de relato de violência física no último ano e $7,4 \%$ durante a gravidez, sendo as formas de agressão mais freqüentes empurrão, tapa e ronchas (manchas roxas) (Tabela 1). Em relação ao padrão da violência, 43,6\% afirmaram ter cessado, 27,3\% diminuído, $18,2 \%$ permanecido inalterado e $10,9 \%$ aumentado durante a gravidez.

Tabela 1 - Distribuição de freqüência das diferentes formas de agressão de que foram vítimas as mulheres que receberam assistência ao parto em uma maternidade de alto risco no período de fevereiro a março de 2001

\begin{tabular}{lcc}
\hline Forma de agressão * & Freqüência & Percentual \\
\hline Empurrão & 31 & 56,4 \\
Tapa & 24 & 43,6 \\
Ronchas (manchas roxas) & 9 & 16,4 \\
Tranco & 5 & 9,1 \\
Bofetada & 4 & 7,3 \\
Murro & 3 & 5,5 \\
Perfurações & 2 & 3,6 \\
\hline
\end{tabular}

*Cada mulher pode ter sido vítima de mais de uma forma de agressão

Analisando-se a associação entre violência física e fatores relacionados à mulher (Tabela 2), verificou-se discreto aumento do risco entre as adolescentes $(25 \%)$, porém sem significância estatística (intervalo de confiança incluindo a unidade). O risco foi significativamente maior para os niveis mais baixos de escolaridade, sendo quase 10 vezes maior para 0-3 anos estudados, em comparação a 11 ou mais anos. Quando se considerou a situação marital, observou-se aumento do 
risco para as mulheres com união consensual em relação às casadas $(77 \%)$, porém essa diferença não foi estatisticamente significante. Não houve diferença na freqüência de violência entre as mulheres com e sem trabalho remunerado, porém o risco foi mais de duas vezes maior entre as tabagistas. A história familiar de violência também apresentou associação estatisticamente significante com a freqüência de violência, implicando risco quase três vezes maior.

Tabela 2 - Risco relativo de violência física no último ano de acordo com fatores relacionados à mulher.

\begin{tabular}{llll}
\hline Variáveis & $\begin{array}{c}\text { Violência } \\
\text { física }\end{array}$ & $\begin{array}{c}\text { Razão de } \\
\text { prevalência }\end{array}$ & IC 95\% \\
& $\mathrm{n} \quad \%$ & \\
\hline
\end{tabular}

\begin{tabular}{|c|c|c|c|c|}
\hline \multicolumn{5}{|l|}{ Idade } \\
\hline $13-19$ anos $(n=132)$ & 20 & 15,2 & 1,25 & $0,75-2,07$ \\
\hline$>20$ anos $(n=288)$ & 35 & 12,2 & 1,0 & \\
\hline \multicolumn{5}{|l|}{ Escolaridade } \\
\hline $0-3$ anos $(n=79)$ & 21 & 26,6 & 9,70 & $2,36-39,94$ \\
\hline 4-7 anos $(n=182)$ & 27 & 14,8 & 5,41 & $1,32-2,19$ \\
\hline $8-10$ anos $(n=86)$ & 5 & 5,8 & 2,12 & $0,42-10,62$ \\
\hline$>11$ anos $(n=73)$ & 2 & 2,7 & 1,0 & \\
\hline \multicolumn{5}{|l|}{ Situação marital } \\
\hline $\begin{array}{l}\text { Sem companheiro } \\
(n=62)\end{array}$ & 6 & 9,7 & 1,11 & $0,40-3,05$ \\
\hline $\begin{array}{l}\text { União consensual } \\
(n=266)\end{array}$ & 41 & 15,4 & 1,77 & $0,86-3,64$ \\
\hline Casadas (n=92) & 8 & 8,7 & 1,0 & \\
\hline \multicolumn{5}{|l|}{ Trabalho remunerado } \\
\hline Não (n=291) & 39 & 13,4 & 1,04 & $0,60-1,79$ \\
\hline $\operatorname{Sim}(n=124)$ & 16 & 12,9 & 1,0 & \\
\hline \multicolumn{5}{|l|}{ Tabagismo } \\
\hline $\operatorname{Sim}(n=49)$ & 12 & 24,5 & 2,11 & $1,20-3,71$ \\
\hline Não (n=370) & 43 & 11,6 & 1,0 & \\
\hline \multicolumn{5}{|l|}{$\begin{array}{l}\text { História familiar de } \\
\text { violência }\end{array}$} \\
\hline $\operatorname{Sim}(n=160)$ & 34 & 21,3 & 2,73 & $1,61-4,61$ \\
\hline Não (n=244) & 19 & 7,8 & 1,0 & \\
\hline
\end{tabular}

Entre os fatores relacionados ao parceiro (Tabela 3), encontrou-se aumento do risco de violência entre adolescentes (50\%), no entanto essa associação não foi significativa. O risco de violência foi significativamente maior para os parceiros com baixa escolaridade (mais de oito vezes) e sem trabalho remunerado (mais de duas vezes). Verificou-se aumento significativo do risco para todos os estratos relativos à embriaguez, quando comparados à ausência: o risco foi mais de três vezes maior quando os parceiros se embriagavam pelo menos uma vez por mês, e quase sete vezes maior para freqüência de embriaguez maior que uma vez por semana. Já em relação à história de violência na família do parceiro, o risco foi quase duas vezes maior entre os homens cujas parceiras relatavam essa história, porém o intervalo de confiança incluiu a unidade.

Tabela 3 - Risco relativo de violência física no último ano de acordo com fatores relacionados ao parceiro.

\begin{tabular}{|c|c|c|c|c|}
\hline \multirow[t]{2}{*}{ Variáveis } & \multicolumn{2}{|c|}{$\begin{array}{l}\text { Violência } \\
\text { física }\end{array}$} & \multirow[t]{2}{*}{$\begin{array}{l}\text { Razão de } \\
\text { prevalência }\end{array}$} & \multirow[t]{2}{*}{ IC $95 \%$} \\
\hline & $\mathrm{n}$ & $\%$ & & \\
\hline \multicolumn{5}{|l|}{ Idade } \\
\hline $13-19$ anos $(n=38)$ & 7 & 18,4 & 1,50 & $0,73-3,08$ \\
\hline$>20$ anos $(n=374)$ & 46 & 12,3 & 1,0 & \\
\hline \multicolumn{5}{|l|}{ Escolaridade } \\
\hline $0-3$ anos $(n=57)$ & 13 & 22,8 & 8,44 & $1,98-35,91$ \\
\hline 4-7 anos $(n=153)$ & 25 & 16,3 & 6,05 & $1,47-24,85$ \\
\hline $8-10$ anos $(n=68)$ & 5 & 7,4 & 2,72 & $0,55-13,56$ \\
\hline$>11$ anos $(n=74)$ & 2 & 2,7 & 1,0 & \\
\hline \multicolumn{5}{|l|}{ Trabalho remunerado } \\
\hline Não (n=181) & 35 & 19,3 & 2,30 & $1,38-3,85$ \\
\hline $\operatorname{Sim}(n=238)$ & 20 & 8,4 & 1,0 & \\
\hline \multicolumn{5}{|l|}{$\begin{array}{l}\text { Freqüência de } \\
\text { embriaguez }\end{array}$} \\
\hline $\begin{array}{l}>1 \text { vez por semana } \\
(n=32)\end{array}$ & 13 & 40,6 & 6,63 & $3,52-12,48$ \\
\hline $\begin{array}{l}1 \text { vez por semana } \\
(n=55)\end{array}$ & 12 & 21,8 & 3,56 & $1,79-7,09$ \\
\hline 1 vez por mês $(n=71)$ & 14 & 19,7 & 3,22 & $1,65-6,27$ \\
\hline Nunca $(n=261)$ & 16 & 6,1 & 1,0 & \\
\hline \multicolumn{5}{|l|}{$\begin{array}{l}\text { História familiar de } \\
\text { violência }\end{array}$} \\
\hline $\operatorname{Sim}(n=160)$ & 13 & 18,1 & 1,92 & $0,99-3,71$ \\
\hline Não (n=24) & 18 & 9,4 & 1,0 & \\
\hline
\end{tabular}

No modelo de regressão logística múltipla (Tabela 4), os fatores que persistiram fortemente associados à violência foram embriaguez do parceiro em qualquer freqüência (risco quase seis vezes maior), ausência de trabalho remunerado do parceiro (risco duas vezes maior), baixa escolaridade e história familiar de violência da mulher (risco mais de três vezes maior).

Quando se analisaram os resultados perinatais de acordo com a presença ou não de violência física doméstica (Tabela 5), não se encontrou associação significativa com prematuridade, baixo peso ao nascer, escores de Apgar no primeiro e quinto minuto e freqüência de natimortos. Por outro lado, a freqüência de neomortos foi mais de quatro vezes maior $(9,1 \%$ versus $2,2 \%$ ) entre as vitimas de violência. 
Tabela 4 - Risco relativo ajustado de violência física no último ano de acordo com fatores relacionados à mulher e ao parceiro (regressão logística múltipla).

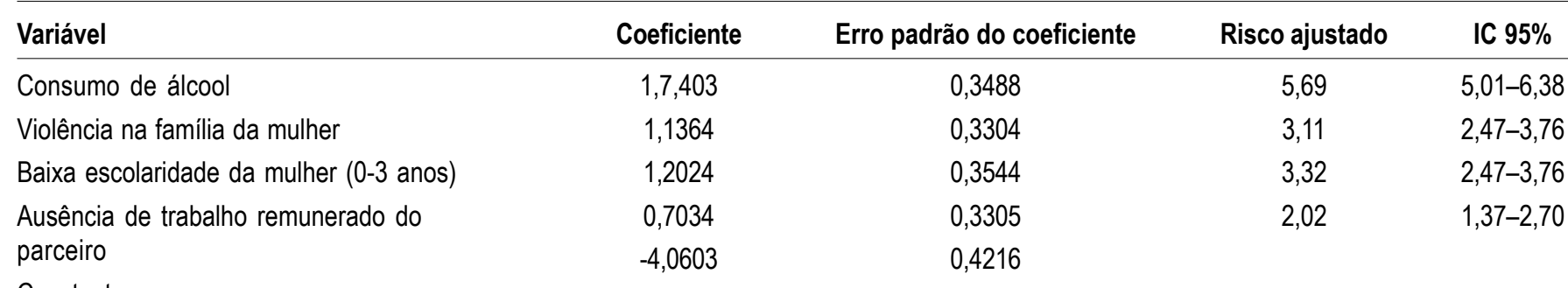

Tabela 5 - Associação entre violência física e resultados perinatais em mulheres que receberam assistência ao parto em uma maternidade de alto-risco em fevereiro e março de 2001

\begin{tabular}{lccccc}
\hline $\begin{array}{l}\text { Resultados } \\
\text { perinatais }\end{array}$ & \multicolumn{2}{c}{$\begin{array}{c}\text { Vítimas de } \\
\text { violência física }\end{array}$} & \multicolumn{2}{c}{$\begin{array}{c}\text { Não-vítimas } \\
\text { de violência }\end{array}$} & $\mathbf{p}$ \\
& $\mathbf{n}$ & $\%$ & $\mathbf{n}$ & $\%$ & \\
\hline Prematuridade & $16 / 55$ & 29,1 & $78 / 359$ & 21,7 & 0,22 \\
Baixo peso ao nascer & $13 / 55$ & 23,6 & $84 / 365$ & 23,0 & 0,92 \\
Apgar < 7 no 10 minuto & $7 / 52$ & 13,5 & $37 / 350$ & 10,6 & 0,53 \\
Apgar < 7 no 50 minuto & $3 / 52$ & 5,8 & $10 / 351$ & 2,8 & 0,3 \\
Natimortos & $2 / 55$ & 3,6 & $11 / 420$ & 3,0 & 0,8 \\
Neomortos & $5 / 55$ & 9,1 & $8 / 365$ & 2,2 & 0,006 \\
\hline
\end{tabular}

\section{Discussão}

Os resultados do presente estudo evidenciam prevalência de violência física antes da gestação em torno de $13 \%$ e durante a gestação de $7,4 \%$. Cerca de $44 \%$ das vítimas referiram que a violência cessou e em torno de $27 \%$ que esta diminuiu, enquanto $18 \%$ informaram padrão inalterado e $10,9 \%$, aumento da violência durante a gravidez.

A prevalência encontrada neste estudo assemelha-se aos resultados descritos por diversos autores, estimando prevalência de violência fisica de $10,9 \%$ antes e $6,6 \%$ durante a gestação ${ }^{26}$, embora outros autores tenham apresentado taxas mais elevadas. No estudo de Canterino et al. ${ }^{27}$, encontrou-se prevalência de violência física de $36 \%$ tanto antes como durante a gravidez, ao passo que Purwar et al. ${ }^{28}$ descreveram taxas de 25 e $22 \%$, respectivamente.

Diferentes prevalências se devem, em primeiro lugar, às características diversas das populações estudadas e, por outro lado, às características metodológicas de cada investigação, referentes tanto ao instrumento de investigação da violência como ao número de vezes que este é aplicado e ao período de investigação em relação à gravidez. Nesse sentido, vale destacar que, em nosso estudo, a entrevista foi realizada em fase crítica do ciclo grávido-puerperal, logo depois do parto, quando as condições psicológicas da mulher podem in- fluenciar fortemente as respostas a qualquer inquérito sobre situação familiar. Prevalências mais elevadas resultam de entrevistas realizadas em mais de uma etapa na gravidez e por profissional de saúde que acompanha o pré-natal ${ }^{15}$.

Apesar dessas considerações em relação a uma possivel subestimativa, ainda assim destacamos que os presentes resultados sobre prevalência de violência física na gravidez apontam para percentuais superiores aos encontrados para outras doenças estudadas na gravidez, no ambulatório de pré-natal, enfermarias de alto risco e setores de assistência obstétrica, tais como o abortamento induzido e o diabete gestacional, aproximando-se bastante da freqüência descrita de hipertensão (em torno de 15\%), uma das maiores complicações gestacionais em nosso meio ${ }^{29}$.

Nessa óptica, justifica-se o interesse em determinar quais os principais fatores de risco associados a maior freqüência de violência contra a mulher, uma vez que a partir de sua identificação podem ser elaboradas estratégias preventivas, visando à redução de sua prevalência. Deve-se ressaltar, outrossim, que uma gama de fatores estão habitualmente inter-relacionados, em uma intricada e perversa seqüência de vitimização da mulher.

Em nosso estudo, a freqüência de violência física foi significativamente maior entre mulheres com baixa escolaridade (zero a três anos estudados), tabagistas e com história familiar de violência, ao passo que as características do parceiro associadas com maior risco foram baixa escolaridade (também zero a três anos estudados), consumo de álcool (embriagar-se pelo menos uma vez por mês) e ausência de trabalho remunerado. $\mathrm{Na}$ análise multivariada, as variáveis que permaneceram mais fortemente associadas ao desfecho de violência física foram a baixa escolaridade e história de violência na família da mulher e consumo de álcool pelo parceiro.

Esses achados são congruentes com a literatura pertinente ao tema, em que se apontam vários dos fatores aqui descritos como associados a risco aumentado de violência. A associação entre violência doméstica e escolaridade, por exemplo, foi já descrita por diversos autores ${ }^{15,30}$, conquanto devamos salientar que os pontos de corte 
para definir o risco de violência encontrem-se bem acima do que foi por nós utilizado, mesmo porque a freqüência de baixa escolaridade é extremamente elevada em nosso país e, sobretudo, em nossa região. A baixa escolaridade das mulheres, bem como dos parceiros, pode determinar dificuldades na relação interpessoal entre homens e mulheres e interferir na resolução de problemas cotidianos, gerando violência.

No que se refere à escolaridade do parceiro, múltiplos fatores interagem mutuamente. A baixa escolaridade interfere nos mecanismos utilizados para a resolução de problemas da mesma forma que na mulher, no entanto há interferência também desta variável no processo de qualificação profissional, resultando em incapacidade para aquisição de condições mínimas de sobrevivência motivadas por salários inapropriados ou por desemprego. Estabelece-se um ciclo vicioso de aumento crescente de tensões, seguido de frustração e agressão. Decorrente desta relação instala-se a violência conjugal e, pela diferença fundamental de força fisica, sofre a mulher as maiores conseqüências.

Por outro lado, mulheres que apresentam história de violência na família, ou seja, testemunharam atos violentos entre seus pais ou substitutos, tendem a apresentar maior tolerância à violência dos seus parceiros, por aceitarem como normalidade formas violentas para a resolução de conflitos, perpetuando o comportamento violento no ambiente familiar ${ }^{31}$. É possivel também que a violência familiar na infância, alterando a estrutura da personalidade e contribuindo para a destruição da auto-estima, permita o exagero da tendência à repetição dos padrões, fazendo com que as mulheres procurem parceiros que se assemelhem, em vários quesitos (consumo de álcool, comportamento violento) aos seus pais agressores.

Associando-se a estes fatores, o álcool parece corroborar a conduta violenta ${ }^{32}$, não de forma direta, mas provavelmente por interferir nos padrões de comportamento facilitando a violência, uma vez que acentua o desequilíbrio de controle e poder exercidos pelo parceiro ${ }^{14,15,24,30}$. No entanto, os reais mecanismos responsáveis por essa associação ainda estão por ser elucidados, não estando claro se o abuso de álcool funciona como um fator causal direto ou indireto, ou ainda como um modificador do efeito de outros fatores ${ }^{30}$.

Já em relação ao tabagismo, que vários autores relatam ser mais freqüente em mulheres vítimas de violência ${ }^{33}$, este parece estar relacionado à tendência da mulher em estabelecer comportamentos de risco para a sua saúde, motivada por fatores relacionados à própria perda da autoestima, que conduzem a progressivo desinteresse no seu bem estar e do seu filho ${ }^{34}$.
Outro aspecto que merece discussão é, como já abordamos, a complexa inter-relação entre os diversos fatores associados à violência contra a mulher. Separar corretamente os verdadeiros fatores de risco das variáveis potencialmente confundidoras exige a adoção de técnicas estatísticas de análise multivariada. Em nosso estudo, realizamos análise de regressão logística múltipla para determinar as variáveis com mais forte associação com a violência física, encontrando um modelo em que as variáveis mais importantes foram escolaridade e história familiar de violência da mulher e abuso de álcool pelo parceiro ${ }^{35}$.

É importante salientar que todos estes fatores fazem parte de um conjunto de situações que se associam e interagem e são resultantes de um ponto único de partida - a precária condição socioeconômica. Familias mais pobres tendem a ser numerosas, gerando incapacidade por parte dos pais de cuidar dos filhos, tanto do ponto de vista de geração de recursos como ainda pela baixo nível de escolaridade. Como resultado surgem a insatisfação e a frustração, que aliadas a comportamentos de risco como o alcoolismo, dão início ao ciclo da violência.

Outro aspecto abordado no presente estudo diz respeito não às causas, mas às conseqüências da violência sobre a gestação e os resultados perinatais. Achado especialmente relevante foi a freqüência de óbitos neonatais na gestação presente, significativamente maior entre as vitimas de violência. Os resultados deste estudo são concordantes com os achados em uma coorte de base hospitalar, com aplicação única de entrevista, na qual o óbito neonatal também mostrou associação com a violência durante a gravide $z^{20}$.

A violência doméstica pode fazer parte de intricada interação de fatores que contribuem para aumento da morbidade e mortalidade perinatais. A proposta de modelo causal para explicar a influência da violência nos resultados perinatais é representada pela interferência de dois mecanismos - um advindo pelo trauma em si, capaz de promover lesões diretas sobre a mulher, que repercutem sobre a gestação, a exemplo do descolamento da placenta, e um segundo mecanismo, baseado na teoria do estresse contínuo ${ }^{36}$.

O estresse contínuo parece afetar o desfecho perinatal por meio de alterações no comportamento dos individuos ou alterando respostas fisiológicas. O comportamento individual pode ser comprometido em diferentes formas, como interferir sobre a capacidade do individuo de manter seu estado nutricional satisfatório, repouso e atendimento à saúde. Paralelamente podem ser assumidos comportamentos de risco que sabidamente interferem nos resultados perinatais, como o tabagismo e uso de drogas lícitas e ilícitas ${ }^{14}$. 
Não obstante todas essas interpretações que pressupomos válidas, devemos apontar algumas limitações metodológicas do presente estudo merecendo discussão. Primeiro, em se tratando de estudo do tipo corte transversal, a relação de causalidade não pode ser determinada. Embora esse desenho de estudo seja adequado para avaliar grandes populações e a determinar a freqüência de determinados eventos, é limitado pelo fato de medir, ao mesmo tempo, exposição e desfecho, de forma que uma ambigüidade temporal dificulta a separação entre causa e efeito ${ }^{30}$.

Acrescenta-se ainda que as características da população estudada, de base hospitalar, não permitem gerar inferências para a população geral. Idealmente, o estudo para avaliar a prevalência de violência doméstica deve ser feito com base populacional ou em setores de assistência primária à saúde. Salientamos, contudo, que em nosso meio, a maioria dos partos são hospitalares, de forma que, se o estudo fosse conduzido em várias maternidades, não haveria motivo para se pensar que a população de puérperas fosse muito diferente das gestantes.

A época em que esses estudos devem ser realizados também merece ser discutida. Acreditamos que caracteristicas peculiares do puerpério podem promover vieses de informação e seleção. É possivel que as mulheres, nessa época, relevem as condutas agressivas do parceiro, na tentativa de manter a relação, principalmente com a chegada de um filho, e, portanto deixem de relatar eventuais episódios de violência. Estabelece-se interiormente uma condição de barganha com o parceiro.

Entretanto, consideramos que o puerpério não deixa de ser época oportuna para investigação de violência, uma vez que para muitas mulheres, representa oportunidade única de contato com o serviço de saúde, com a vantagem de permitir condições para avaliação da violência durante toda a gravidez, já que pode haver modificação dos padrões de violência durante a gestação. Em relação aos vieses de informação, o que se pode obter é resultado subestimado, que mesmo assim chama a atenção para a gravidade do problema, porque se há erro, é erro para menos, e certamente a prevalência poderia ter sido maior do que a encontrada.

Apesar destas limitações, inerentes a qualquer estudo de prevalência, devemos destacar a importância do presente estudo que, até aonde chega nosso conhecimento, representa o primeiro estudo brasileiro sobre violência em gestantes e é indiscutivelmente pioneiro em nossa região e em nosso serviço. Acreditamos que, além de sensibilizar os profissionais de saúde para o problema, nossos resultados, e particularmente os da- dos epidemiológicos do estudo, poderão ser utilizados com a finalidade de estabelecer vínculos entre a violência e as situações especiais da gravidez. Avaliar fatores de risco para a violência pode facilitar seu diagnóstico e permitir ações médicas que determinem menor óbice sobre a gestação e o produto conceptual, porque, como demonstramos, a mortalidade neonatal encontra-se aumentada entre as vítimas de violência.

\section{ABSTRACT}

Purpose: to determine the prevalence of domestic physical violence among women who delivered at a tertiary center in the Northeast of Brazil, to study the main risk factors associated with domestic violence, and to determine perinatal outcome. Methods: a cross-sectional study was conducted, enrolling 420 women who delivered at a tertiary center in Recife (Brazil) with fetuses weighing more than $500 \mathrm{~g}$. They were submitted to interviews with open and closed questions. The prevalence of domestic physical violence was determined. Statistical analysis was performed using $\chi^{2}$ and Fisher's exact tests at a 5\% level of significance. The prevalence ratio was determined as measurement of relative risk of violence. Multiple logistic regression analysis was performed and the adjusted risk was calculated.

Results: the prevalence of domestic physical violence was $13.1 \%(95 \% C I=10.1-16.6)$ and $7.4 \%(95 \% C I=5.2-10.2)$ before and during pregnancy, respectively. The pattern of violence has changed during pregnancy: stopped in $43.6 \%$, was reduced in $27.3 \%$ and increased in $11 \%$ of the victims. After multivariate analysis the variables that persisted strongly associated with violence were low female educational level, history of violence in the women's family, partner's use of alcohol and unemployment. Perinatal outcome was studied and a significantly higher frequency of neonatal death was observed among victims of domestic violence.

Conclusions: a high prevalence of domestic physical violence was observed (about 13\%) in women who delivered at a tertiary center in Northeast of Brazil. The main risk factors were low educational level and previous familiar history of violence in the women's family, alcohol use by and unemployment of their partners. Neonatal mortality was increased in victims of violence.

KEYWORDS: Violence against women. Domestic violence. Pregnancy. Violence and pregnancy: risk factors.

\section{Referências}

1. World Health Organization. Violence against women: a priority health issue. 1997. Available from: URL: http://www.who.int/violence_injury_prevention/ vaw/ infopack.htm

2. El-Bayoumi G, Borum ML, Haywood Y. Domestic violence in women. Med Clin North Am 1998; 82:391-401. 
3. Ellsberg M, Peña R, Herrer A, Liljestrand J, Winkvist, A. Candies in hell: women's experiences of violence in Nicaragua. Soc Sci Med 2000; 51:1595-610.

4. Haile-Mariam T, Smith J. Domestic violence against women in the international community. Emerg Med Clin North Am 1999; 17:617-30.

5. Eilenberg $J$, Fullilove $M$. Introduction. In: Commonwealth Fundation Commission on Women's Health. Violence Against Women in the United States: a comprehensive background paper. $2^{\text {nd }}$ ed. New York: The Commission; 1996. p. 1-5.

6. Heise L, Pitanguy A, Germain A. Violence against women: the hidden health burden. $1^{\text {st }}$ ed. Washington, DC: The World Bank; 1994.

7. American College of Obstetrics and Gynecologists. Domestic violence. Washington, DC: ACOG; 1999. (ACOG Technical Bulletin, 257).

8. Rodriguez MA, Bauer HM, McLoughlin E, Grumbach $K$. Screening and intervention for intimate partner abuse: practices and attitudes of primary care physicians. JAMA 1999; 282:468-74.

9. Wilt S, Olson S. Prevalence of domestic violence in the United States. J Am Med Womens Assoc 1996; $51: 77-83$.

10.National Survey of Women's Health. Survey of women's health highlights. 1993. Available from: URL: http://ww.cmwf.org/programs/women / whhilite.asp

11. Chambliss LR. Domestic violence: a public health crisis. Clin Obstet Gynecol 1997; 40:630-8.

12.Leung WC, Leung TW, Lam YY, Ho PC. The prevalence of domestic violence against pregnant women in a Chinese community. Int $\mathrm{J}$ Gynaecol Obstet 1999; 66:23-30.

13. Hamberger LK, Ambuel B. Spousal abuse in pregnancy. Clin Fam Pract 2001; 3:203-24.

14.Hedin LW, Janson PO. Domestic violence during pregnancy. The prevalence of physical injuries, substance use, abortions and miscarriages. Acta Obstet Gynecol Scand 2000; 79:625-30.

15.Gazmararian JA, Petersen R, Spitz AM, Goodwin MM, Saltzman LE, Marks JS. Violence and reproductive health: current knowledge and future research directions. Matern Child Health J 2000; 4:79-84.

16. Moore M. Reproductive health and intimate partner violence. Fam Plann Perspect 1999; 31:302-12.

17.Faúndes A, Hardy E, Osis MJ, Duarte G. O risco para queixas ginecológicas e disfunções sexuais segundo história de violência sexual. Rev Bras Ginecol Obstet 2000; 22:153-7.

18.Dietz PM, Spitz AM, Anda RF, et al. Unintended pregnancy among adult women exposed to abuse or household dysfunction during their childhood. JAMA 1999; 282:1359-64.

19.Parker B, McFarlane J, Soeken K. Abuse during pregnancy: effects on maternal complications and birth weight in adult and teenage women. Obstet Gynecol 1994; 84:323-8.
20.Webster J, Sweett S, Stolz TA. Domestic violence in pregnancy. A prevalence study. Med J Aust 1994; 161:466-70.

21.Berenson AB, Wiemann CM, Wilkinson GS, Jones WA, Anderson GD. Perinatal morbidity associated with violence experienced by pregnant women. Am J Obstet Gynecol 1994; 170:1760-6.

22.Jejeebhoy SJ. Associations between wife-beating and fetal and infant death: impressions from a survey in rural India. Stud Fam Plann 1998; 29:300-8.

23. Coker AL, Smith PH, McKeown RE, King MJ. Frequency and correlates of intimate partner violence by type: physical, sexual, and psychological battering. Am J Public Health 2000; 90:553-9.

24.Datner EM, Ferroggiaro AA. Violence during pregnancy. Med Clin North Am 1999; 17:645-56.

25.Reichenhein ME, Moraes CL, Hasselmann $\mathrm{MH}$. Equivalência semântica da versão em português do instrumento "Abuse Assessment Screen" (AAS) para rastrear a violência contra a mulher grávida. Rev Saúde Pública 2000; 34:610-6.

26. Stewart DE, Cecutti A. Physical abuse in pregnancy. CMAJ 1993; 149:1257-63.

27.Canterino JC, VanHorn LG, Harrigan JT, Ananth CV, Vintzileos AM. Domestic abuse in pregnancy: a comparison of a self-completed domestic abuse questionnaire with a directed interview. Am J Obstet Gynecol 1999; 181:1049-51.

28.Purwar MB, Jeyaseelan L, Varhadpande U, Motghare V, Pimplakute S. Survey of physical abuse during pregnancy GMCH, Nagpur, India. J Obstet Gynaecol Res 1999; 25:165-71.

29.Santos LC, Porto AMF, Amorim MMR, Guimarães V. Atualização - Obstetrícia: diagnóstico e tratamento. $1^{\mathrm{a}}$ ed. Recife: IMIP; 2000. v.2.

30.Kyriacou DN, Anglin D, Taliaferro E, et al. Risk factors for injury to women from domestic violence against women. N Engl J Med 1999; 341:1892-8.

31.Buvinic M, Morrison A, Shifter M. La violencia en América Latina y el Caribe: un marco de referencia para la acción. $1^{\mathrm{a}}$ ed. Washington, DC: Banco Interamericano de Desarrollo; 2000.

32.Corsi J. Violencia familiar: una mirada interdisciplinaria sobre un grave problema social. $1^{\mathrm{a}}$ ed. Buenos Aires: Paidos; 1994. p.252.

33.Grimstad H, Schei B, Backe B, Jacobsen G. Physical abuse and low birthweight: a case control study. $\mathrm{Br}$ J Obstet Gynaecol 1997; 104:1281-7.

34.Bhatt RV. Domestic violence and substance abuse. Int J Gynaecol Obstet 1998; 63 (Suppl 1):S25-31.

35.Kunitz SJ, Levy JE, McCloskey J, Gabriel KR. Alcohol dependence and domestic violence as sequelae of abuse and conduct disorder in childhood. Child Abuse Negl 1998; 22:1079-91.

36. Petersen R, Gazmararian JA, Spitz AM, et al. Violence and adverse pregnancy outcomes: a review of the literature and directions for future research. Am J Prev Med 1997; 13:366-73.

Recebido em: 14/2/2003 Aceito com modificações em: 8/5/2003 